\title{
ANDES

www.scielo.cl

\section{Manejo de enfisema intersticial unilateral adquirido en un recién nacido prematuro mediante intubación bronquial selectiva: descripción de un caso y revisión de la literatura}

\section{Management of unilateral acquired pulmonary bullous emphysema in a premature infant through selective bronchial intubation. Case report and review of literature}

\author{
Patricia Álvarez C. a,b, Mauricio Marín R. ${ }^{\mathrm{a}}$
}

aservicio de Neonatología, Hospital de Puerto Montt. Puerto Montt, Chile

bUniversidad San Sebastián Sede de la Patagonia. Puerto Montt, Chile

Recibido: 3 de septiembre de 2020; Aceptado: 30 de noviembre de 2020

¿Qué se sabe del tema que trata este estudio?

El enfisema intersticial bulloso unilateral adquirido es una patología poco frecuente desde el advenimiento del surfactante y la ventilación gentil. Es importante reconocerla y puede tratarse exitosamente con intubación selectiva del pulmón contralateral cuando fallan otras medidas terapéuticas
¿Qué aporta este estudio a lo ya conocido?

La descripción detallada del procedimiento y consideraciones que se deben tener presente para que la técnica sea exitosa

\section{Resumen}

El enfisema bulloso adquirido unilateral es una patología poco frecuente del recién nacido, de difícil manejo ventilatorio. Objetivo: Describir un caso que requirió de intubación bronquial selectiva del pulmón contralateral y revisión de la literatura. Caso Clínico: recién nacido prematuro de 27 semanas que evoluciona con enfisema bulloso unilateral izquierdo secundario a una enfermedad por déficit de surfactante. El paciente no respondió a las medidas habituales de posicionamiento, disminución de presiones inspiratorias máximas, frecuencia respiratoria y tiempo inspiratorio. Evolucionó grave, con requerimiento de oxígeno elevado, cada vez mayor distensión del pulmón afectado y atelectasia del pulmón contralateral. Se realizó monointubación del pulmón derecho, siendo el procedimiento bien tolerado. A las 48 horas se observó el pulmón afectado completamente colapsado y el contralateral adecuadamente insuflado, por lo que se decidió ventilar ambos pulmones nuevamente. Evolucionó favorablemente, lográndose extubar a los 6 días del procedimiento y fue dado de alta con radiografía normal, sin observarse imágenes quísticas. Conclusiones: La monointubación selectiva ha demostrado ser segura y eficiente en casos de enfisema bulloso unilateral cuando no hay respuesta a las medidas habituales.
Palabras clave: Enfisema Bulloso; Monointubación; Intubación Selectiva; Enfisema Intersticial Pulmonar 


\begin{abstract}
Acquired pulmonary bullous emphysema is an infrequent complication of assisted ventilation in the premature infant that is difficult to manage. Objective: The goal of this report is to present the case of a premature infant who required selective bronchial intubation as well as to provide a review of the current literature on the subject. Clinical Case: The patient is a 27-week gestational age neonatal female patient whose clinical course was complicated by left unilateral bullous emphysema during assisted ventilation for respiratory distress syndrome. Lower peak inspiratory pressures, higher respiratory frequencies, patient positioning, and lower inspiration time failed to improve the patient's condition. The left lung became critically overinflated and compressed the right lung to the point of atelectasis. The patient was selectively mono intubated through the right main bronchus, which resulted in a collapse of the left emphysematous lung. Single right lung ventilation was continued for 48 hours before restarting conventional ventilation of both lungs. Our patient improved significantly, was extubated 6 days after the procedure and later discharged home with normal chest $\mathrm{x}$-ray images. Conclusion: Selective bronchial intubation is a safe and effective procedure in cases of acquired bullous emphysema when usual ventilatory management fails.
\end{abstract}

\section{Keywords:}

Bullous Emphysema; Selective Intubation; Pulmonary Interstitial Emphysema

\section{Introducción}

El enfisema intersticial y bulloso es una complicación cada vez menos frecuente gracias al uso de corticoides antenatales, administración de surfactante y técnicas ventilatorias que protegen al pulmón ${ }^{1}$. Cuando el enfisema bulloso es unilateral puede causar sobredistensión progresiva del lado afectado y atelectasia del pulmón contralateral por compresión, que lleva a un cuadro difícil de manejar desde el punto de vista ventilatorio $^{2}$ y aumenta el riesgo de muerte. Cuando las medidas terapéuticas habituales (disminuir tiempo y presiones inspiratorias, posicionamiento, ventilación de alta frecuencia, administración de corticoides) fallan, la alternativa de intubación selectiva del pulmón contralateral rompe el círculo vicioso y da tiempo para que se recupere el pulmón con enfisema ${ }^{3}$. El objetivo de este artículo es presentar el caso de un paciente con enfisema bulloso unilateral que evolucionó grave y se benefició de intubación bronquial selectiva.

\section{Caso Clínico}

Recién nacido pretérmino de 27 semanas, peso $1251 \mathrm{~g}$, grande para edad gestacional, producto de embarazo gemelar bicorial biamniótico. Madre multípara de 5 con antecedente de ITU reciente por Ureaplasma urealyticum tratada, diabetes gestacional y usuaria de pesario. La madre se hospitalizó por síntomas de parto prematuro, recibiendo una dosis de ampicilina de $2 \mathrm{~g}$, una carga de sulfato de magnesio e indometacina. Recibió una dosis de corticoides antenatal 8 horas previas al parto. APGAR 4-7, requiriendo ventilación a presión positiva con tubo de reanimación en $\mathrm{T}$ con PIP de 20 y PEEP de $6 \mathrm{~cm} \mathrm{H}_{2} \mathrm{O}$ con $\mathrm{FiO}_{2}$ hasta $100 \%$, por lo que se intubó en sala de partos con tubo 2,5 a $7 \mathrm{~cm}$ en comisura bucal. Ingresó a UCI con $\mathrm{FiO} 2$ de $60 \%$, se conectó a ventilación mecánica SIMV 18/5, Tim 0,33, FR: 30/min y se iniciaron antibióticos de primera línea. El recién nacido evolucionó con enfermedad por déficit de surfactante (figura 1) que requirió de 3 dosis de Survanta ${ }^{\circledR}$, a los 45 min de vida, 9 y media horas y a las 36 horas de vida.

A pesar del surfactante, al segundo día de vida presentó mayor compromiso respiratorio, por lo que se sospechó posible neumonía y se cambiaron antibióticos a cefotaxima más cloxacilina. Ese mismo día presentó neumotórax a tensión bilateral que requirió de instalación de drenaje pleural a ambos lados (figura 2).

Pese al cambio de antibióticos, aumentó el compromiso respiratorio requiriendo ventilación de alta frecuencia con $\mathrm{FiO}_{2}$ de $100 \%$ y presión media de vía aérea de hasta $13 \mathrm{cmH}_{2} \mathrm{O}$ desde el tercer día de vida. Posteriormente presentó tres episodios de neumotórax

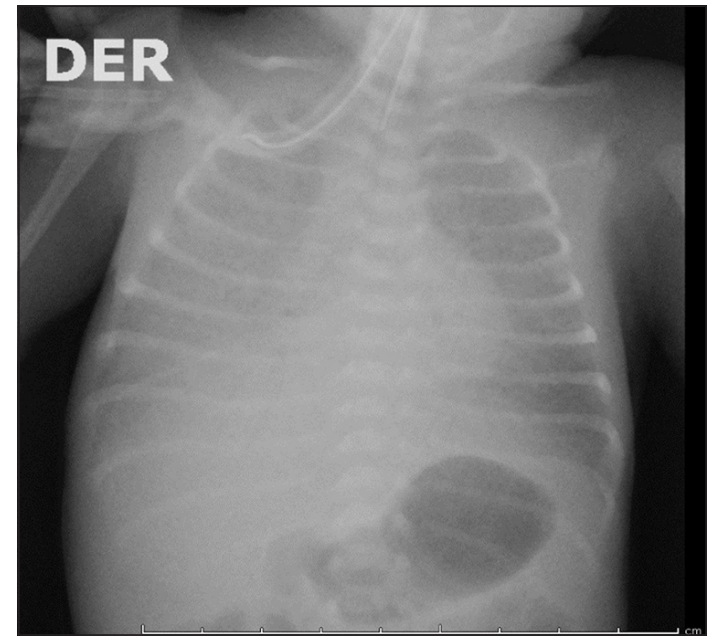

Figura 1. Primera radiografía a los 40 min de vida que muestra imagen compatible con enfermedad por déficit de surfactante. 

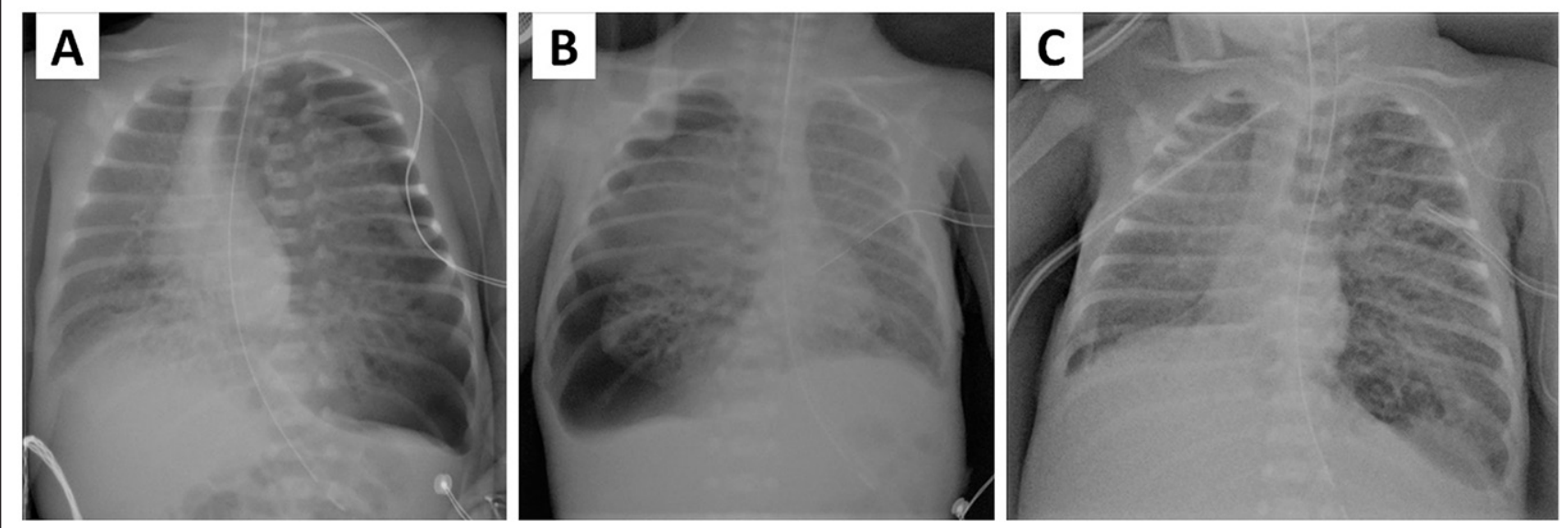

Figura 2. Secuencia de radiografías, todas del día 3 donde se observa neumotórax izquierdo (A) y luego neumotórax derecho (B) con instalación de drenaje a ambos lados (C). En la última imagen además se puede observar inicio de áreas de enfisema intersticial pulmonar izquierdo.

izquierdo, llegando a estar con dos tubos de drenaje pleural en ese hemitórax. Radiografías de control mostraron cada vez mayor hiperinsuflación del pulmón izquierdo junto a enfisema intersticial difuso y aparición de bulas en base izquierda (figura 3). El manejo respiratorio resultó especialmente difícil, incluso con el paciente en decúbito lateral sobre el lado enfisematoso (izquierdo), no logrando parámetros adecuados en ventilación mecánica que frenaran la hiperexpansión pulmonar izquierda.

El día 13 de vida, el paciente se encontraba grave y comprometido del punto de vista respiratorio, requiriendo $\mathrm{FiO}_{2}$ de $100 \%$, con enfisema intersticial y bulloso de pulmón izquierdo cada vez más severo que comprimía el pulmón derecho, por lo que se decidió monointubar a derecha. El procedimiento se realizó en ventilación convencional modo asistido controlado con PIM/PEEP de $17 / 5 \mathrm{cmH}_{2} \mathrm{O}$, FR: $45 / \mathrm{min}$, Ti: 0,35 seg; luego se introdujo el tubo (el mismo que tenía desde el nacimiento) hasta que se observó disminución del murmullo pulmonar a izquierda siempre conectado al ventilador mecánico (no se usó bolsa autoinflable durante el procedimiento). En el momento de la máxima profundidad de introducción del tubo, el paciente presentó episodio de desaturación que mejoró rápidamente al retirar levemente el tubo, quedando finalmente $2 \mathrm{~cm}$ más introducido de lo que estaba previo al procedimiento (figura 4 ).

Luego de la monointubación se mantuvo ventilación con objetivo de presión y el paciente evolucionó estable, los requerimientos de $\mathrm{FiO}_{2}$ disminuyeron y los gases arteriales mostraron mejoría de $\mathrm{pH}$ y $\mathrm{pCO}_{2}$ (tabla 1). Las radiografías de control mostraron adecuada expansión de pulmón derecho y colapso completo del pulmón izquierdo (figura 5). Luego de $48 \mathrm{~h}$ de monointubación se retiró el tubo, reiniciando ventilación

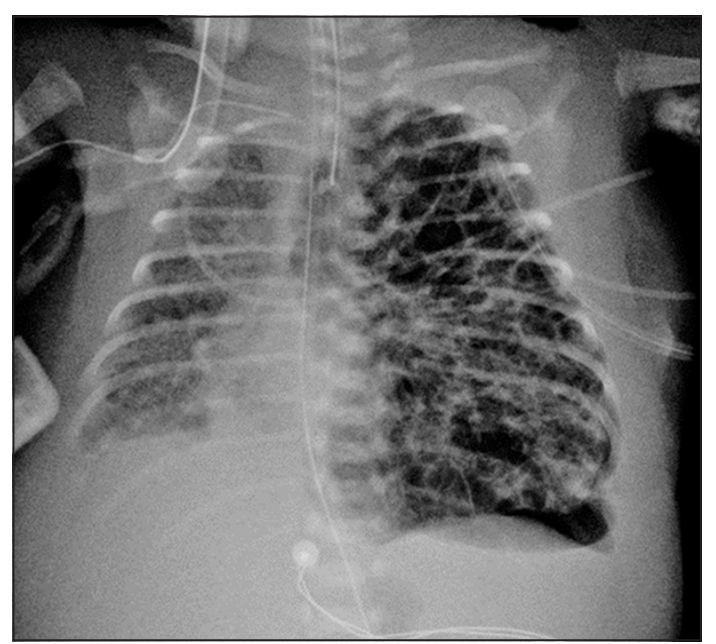

Figura 3. Se observa hiperinsuflación severa de pulmón izquierdo secundaria a enfisema intersticial bulloso que colapsa pulmón contralateral.

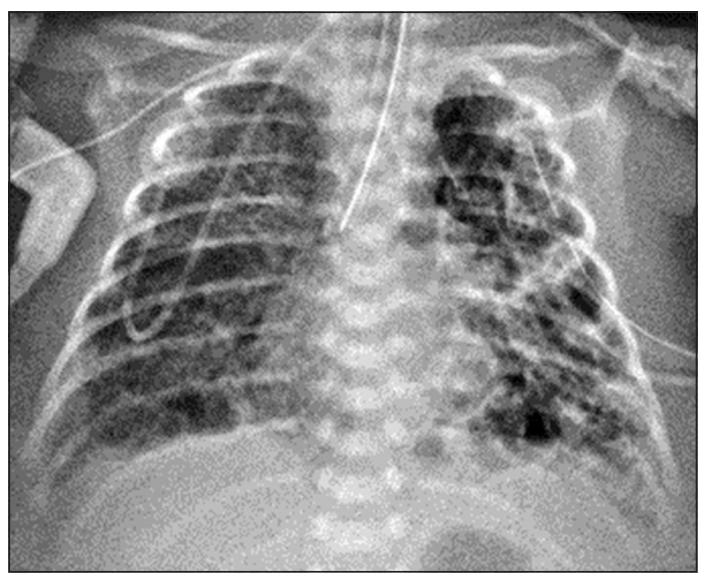

Figura 4. Primera radiografía luego de monointubación del pulmón derecho. Se observa inmediatamente una mejoría de la distensión del pulmón izquierdo. 
Tabla 1. Evolución de $\mathrm{FiO}_{2}, \mathrm{pH}, \mathrm{pCO}_{2}$ e índice de oxigenación

\begin{tabular}{lcccc}
\hline Parámetro & $\begin{array}{c}\text { Previo a } \\
\text { monointubación }\end{array}$ & $\begin{array}{c}6 \text { h post } \\
\text { monointubación }\end{array}$ & $\begin{array}{c}\text { 48 h post } \\
\text { monointubación }\end{array}$ & $\begin{array}{c}24 \mathrm{~h} \text { suspendida } \\
\text { monointubación }\end{array}$ \\
\hline $\mathrm{FiO}_{2}(\%)$ & 100 & 95 & 64 & 60 \\
$\mathrm{pH}$ & 7,174 & 7,204 & 7,377 & 7,471 \\
$\mathrm{pCO}_{2}(\mathrm{mmHg})$ & 83,6 & 75,5 & 47,0 & 37,1 \\
$\mathrm{IO}$ & 22,8 & 16,9 & 10,8 & 6,8 \\
\hline
\end{tabular}

$\mathrm{FiO}_{2}$ : fracción inspirada de oxigeno; $\mathrm{pCO}_{2}$ : presión parcial de $\mathrm{CO}_{2} ; \mathrm{IO}$ : índice de oxigenación.

de ambos pulmones y las radiografías de control mostraron una buena expansión de ambos campos. A los 6 días de suspendida la monointubación se extubó a CPAP. A los 30 días de vida se suspendió CPAP y pasó a cánula nasal de alto flujo a $4 \mathrm{l} / \mathrm{min}$, a los 48 días inicia naricera a $0,1 \mathrm{l} / \mathrm{min}$ y a los 46 días queda respirando aire ambiental. Se realizó saturometría nocturna con aire ambiental que fue informada como normal. La radiografía de tórax previo al alta mostró campos pulmonares simétricos sin evidencias de enfisema ni bulas (figura 6).

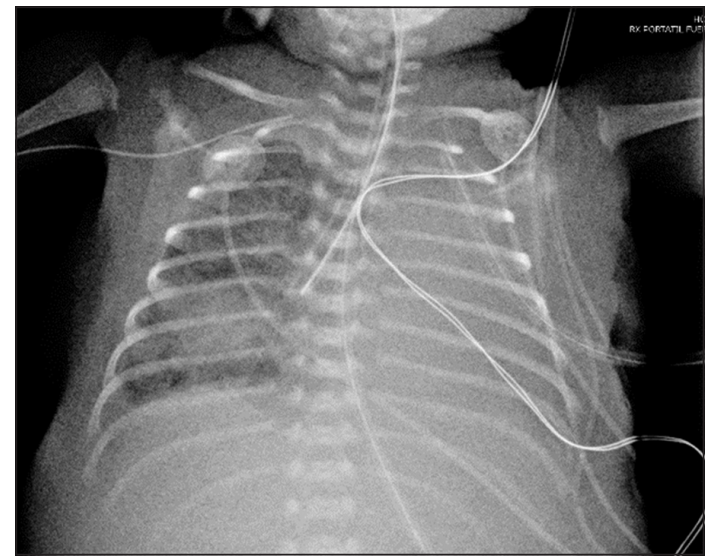

Figura 5. Colapso completo de pulmón izquierdo y mejor insuflación pulmón derecho 48 h posterior a monointubación

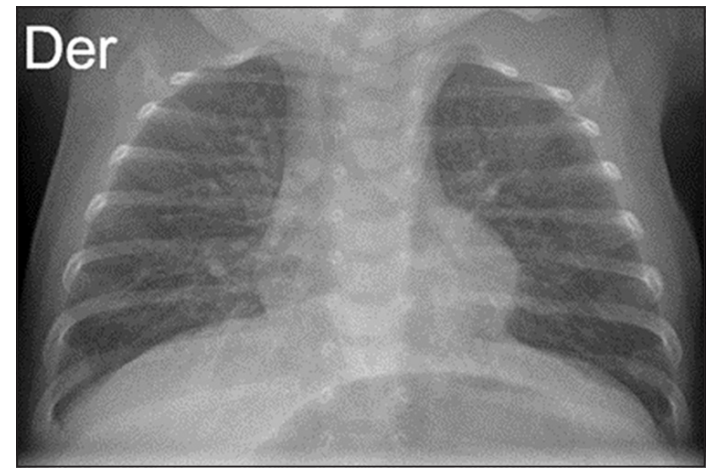

Figura 6. Radiografía previo al alta. Se observan ambos campos pulmonares simétricos, sin bulas.

\section{Discusión}

El escape aéreo es una complicación de la enfermedad por déficit de surfactante que afecta hasta un $10 \%$ de los prematuros con apoyo ventilatorio. El enfisema pulmonar intersticial y el enfisema bulloso adquirido son tipos de escapes aéreos ${ }^{4,5}$. El enfisema intersticial corresponde a ruptura alveolar y acumulación de aire en el compartimiento intersticial. La falta de poros de Kohn en un pulmón inmaduro puede contribuir a esta patología en un recién nacido prematuro ${ }^{3}$. Si la cantidad de aire intersticial es significativa, puede separar parénquima pulmonar adyacente, formando enfisema bulloso de tamaño variable ${ }^{2,4}$. Se relaciona el desarrollo de enfisema intersticial a presiones inspiratorias y $\mathrm{FiO} 2$ elevadas, especialmente dentro de las primeras 24 horas $^{1}$. A pesar de la administración del surfactante y de técnicas más modernas de ventilación, el daño pulmonar por barotrauma puede ser reducido, pero no completamente evitado ${ }^{6}$. El enfisema intersticial y bulloso altera la ventilación-perfusión del pulmón afectado y lleva a la aparición de neumotórax, neumomediastino y está asociado a mayor riesgo de muerte y displasia broncopulmonar ${ }^{34}$.

El enfisema intersticial puede ser unilateral o bilateral. En caso de que sea unilateral, puede causar atelectasia del pulmón contralateral por compresión, necesidad de presiones ventilatorias más altas, sobredistensión progresiva del lado afectado que genera un círculo vicioso que puede llevar a una falla catastrófica de la ventilación, como en el caso presentado ${ }^{2}$. El manejo terapéutico del enfisema bulloso es difícil y no siempre exitoso. Dentro de las opciones están posicionamiento del neonato sobre el lado afectado, disminuir presiones inspiratorias máximas, disminuir frecuencia respiratoria y acortar el tiempo inspiratorio ${ }^{7,8}$. Otras medidas terapéuticas incluyen el uso de ventilación de alta frecuencia, administración de corticoides, resección quirúrgica de la porción más afectada e intubación selectiva del pulmón contralateral ${ }^{5}$.

La intubación selectiva del pulmón atelectasiado rompe el círculo vicioso y da tiempo para que se recupere el enfisema intersticial con menos barotrauma ${ }^{3}$. La mayoría de los casos publicados han usado la mo- 
nointubación por 1 a 7 días antes de ventilar nuevamente ambos campos pulmonares, pero algunos autores aconsejan un mínimo de $48 \mathrm{~h}$ para disminuir riesgo de recurrencia ${ }^{2,5,7,9}$. Una monointubación mayor de 6 días puede llevar a atelectasias del pulmón no ventilado, bradicardia, episodios de desaturación y neumonías ${ }^{7}$. Según una serie de 9 casos publicados por André Jakob et al, la mayoría de los pacientes no presentaron recurrencia del cuadro luego de ventilar ambos campos pulmonares ${ }^{2}$. Observaron que, si se produce una atelectasia completa del lóbulo afectado durante la monointubación por tiempo mayor de 48 h, es más probable que el enfisema bulloso se resuelva permanentemente. En el paciente presentado, se decidió ventilar ambos pulmones a las 48 horas, debido a que el pulmón afectado se había colapsado completamente y el pulmón contralateral se observaba adecuadamente expandido.

El objetivo de la monointubación es lograr una vasoconstricción hipóxica, dejando al pulmón excluido no solo de la ventilación, sino también de la perfusión. Para esto, la monointubación debe lograr un buen selle y lograr el colapso completo del pulmón afectado, de esta forma disminuye al mínimo la alteración de la ventilación/perfusión y se evita hipercapnia e hipoxia ${ }^{10}$.

En caso de monointubar el pulmón derecho, si se introduce demasiado el tubo endotraqueal, se ocluye la ventilación del lóbulo superior derecho, por esta razón, la ubicación debe ser inmediatamente distal a la carina y con la abertura del bisel hacia el lado que se desea monoventilar ${ }^{2,11}$. Se recomienda ajustar la posición del tubo y retirarlo ligeramente si en la radiografía de control se observa colapso del lóbulo superior o medio derecho ${ }^{11}$. En nuestro caso, la desaturación que presentó el paciente al alcanzar el tubo la máxima profundidad, podría haber sido originada por la oclusión del lóbulo medio y superior derecho, que mejoró al ajustar la posición del tubo.

Se piensa que la ventilación de alta frecuencia podría ser más indicada al ventilar un solo pulmón, ya que puede oxigenar y ventilar mejor con presiones medias de vía aérea relativamente más bajas, aunque en algunas publicaciones manejaron al paciente en ventilación mecánica convencional con buenos resultados, aconsejando como medidas protectoras pulmonares, mantener PEEP entre 4-6 $\mathrm{cmH}_{2} \mathrm{O}$ y usar volúmenes tidales bajos ${ }^{2,6,11,12}$. En nuestro caso, el paciente respondió bien a ventilación mecánica convencional con objetivo de presión.

En una revisión de 40 casos hecha por Joseph et al., el 74\% tenía afectado el pulmón izquierdo, requiriendo de intubación selectiva del pulmón derecho, como en nuestro caso ${ }^{9}$. En esa revisión se encontró un 52\% de complicaciones durante el procedimiento e incluyeron malposición del tubo, bradicardia, hipoxia y ate- lectasia del lóbulo superior derecho; todas estas fueron informadas como complicaciones breves y reversibles. Se informó de tres pacientes fallecidos, uno debido a hemorragia intraventricular, otro debido a neumopericardio poco después del procedimiento y el tercero por una neumonía del pulmón no ventilado. Los autores aconsejan que de ser necesario el procedimiento, esperar hasta al menos el séptimo día de edad, para disminuir riesgo de hemorragia intraventricular.

Cuando el enfisema bulloso es en el pulmón derecho, la monointubación del pulmón izquierdo es técnicamente más difícil debido al ángulo del bronquio fuente izquierdo $\left(49^{\circ}\right.$ comparado con $31^{\circ}$ del bronquio fuente derecho). Chalak et al. describen una técnica para intubar selectivamente el bronquio izquierdo usando sólo laringoscopio y tubo endotraqueal, que, en caso de fallar, se puede recurrir a intubación guiada por broncoscopía u obstrucción del bronquio fuente derecho mediante catéter vascular con balón; dejando la oclusión con balón como última alternativa por el riesgo de lesionar la mucosa bronquial y producir estenosis ${ }^{7,10,13,14}$.

\section{Conclusiones}

El enfisema bulloso es un tipo de escape aéreo cada vez menos frecuente, pero que cuando se presenta y es unilateral puede ser de difícil manejo del punto de vista ventilatorio. La monointubación del pulmón contralateral ha demostrado ser un método terapéutico eficiente cuando fallan otras alternativas de manejo. En caso de requerirse, se debe considerar al menos $48 \mathrm{~h}$ de monointubación para disminuir la probabilidad de recurrencia del cuadro.

\section{Responsabilidades Éticas}

Protección de personas y animales: Los autores declaran que los procedimientos seguidos se conformaron a las normas éticas del comité de experimentación humana responsable y de acuerdo con la Asociación Médica Mundial y la Declaración de Helsinki.

Confidencialidad de los datos: Los autores declaran que han seguido los protocolos de su centro de trabajo sobre la publicación de datos de pacientes.

Derecho a la privacidad y consentimiento informado: Los autores han obtenido el consentimiento informado de los pacientes y/o sujetos referidos en el artículo. Este documento obra en poder del autor de correspondencia.

\section{Conflicto de intereses}

Los autores declaran no tener conflicto de intereses. 


\section{Referencias}

1. Ferraz Liz C, Novo A, Nascimento M, Ferreira-Magalhaes M, Carvalho C, Proença E. Selective lung ventilation in the management of unilateral pulmonary interstitial emphysema. J Paediatr Child Health. 2020;56:1461-3.

2. Jakob A, Bender C, Henschen M et al. Selective Unilateral Lung Ventilation in Preterm Infants with Acquired Bullous Emphysema: A Series of Nine Cases. Pediatr Pulmonol. 2013;48:14-9.

3. Khashu M, Mawson J, Bhargava S, Jeet Singh A. A newborn requiring selective bronchial intubation. CMAJ 2005; 173:872.

4. Mühlhausen G, Brethauer S, Martínez F, Melipillán Y. Enfisema pulmonar intersticial persistente en recién nacido. Caso clínico. Rev Chil Pediatr. 2012;83:73-7.

5. Andreou A, Papouli M, Netskos D, Papadopoulou F, Rossiou E. One-Sided High-Frequency Oscillatory Ventilation in the Management of an
Acquired Neonatal Lobar Enphysema: A Case Report and Review. J Perinatol. 2001;21:61-4.

6. Rettwitz-Volk W, Schlösser R, von Loewenich V. One-sided high-frequency oscillating ventilation in the treatment of neonatal unilateral pulmonary emphysema. Acta Paediatr. 1993;82: 190-2.

7. Chalak L, Kaiser J, Arrington R. Resolution of pulmonary interstitial emphysema following selective left main stem intubation in a premature newborn: an old procedure revisited. Pediatric Anesthesia 2007;17:183-6.

8. Lei X, Stangl O, Bösche C, Stuchlik K, Czorba R, Wieg C. Positional treatment without mechanical ventilation in a very preterm infant with unilateral pulmonary interstitial emphysema: case report and review of the literature. BMC Pediatr. 2019;19:267-71.

9. Joseph L, Bromiker R, Toker O, Schimmel M, Goldberg S, Picard E. Unilateral lung intubation for pulmonary air leak syndrome in neonates: a case series and a review of the literature. Am J Perinatol. 2011;28:151-6.

10. Marraro G. Selective bronchial intubation for one-lung ventilation and independentlung ventilation in pediatric age: state of the art. Chin J Contemp Pediatr. 2020;22:543-54.

11. Lee S R. Application of Selective Bronchial Intubation versus Neurally Adjusted Ventilatory Assist in the Management of Unilateral Pulmonary Interstitial Emphysema: An Illustrative Case and the Literature Review. Am J Perinatol Rep. 2017;7:101-5.

12. Liu J, Liao X, Li Y et al. Effect of low tidal volume with PEEP on respiratory function in infants undergoing one-lung ventilation. Anaesthesist 2017;66:667-71.

13. Hathorn C, Armitage N, Wensley D, Seear M. Bronchial balloon occlusion in children with complex pulmonary air leaks. Arch Dis Child. 2013;98:136-40.

14. Jakob A, Hentschel R. Air leaks and pulmonary emphysema in infants: selective bronchial intubation or balloon occlusion? Arch Dis Child. 2013;98:649. 